\title{
Ways of Increasing Synergy in Automated Factory Design and Commissioning Teamwork
}

\author{
Rommi Källo, Martin Eerme and Vello Reedik \\ Department of Machinery, Tallinn University of Technology, Ehitajate Ttee 5, Tallinn 19086, Estonia
}

Received: August 25, 2013 / Accepted: September 10, 2013 / Published: September 25, 2013.

\begin{abstract}
The present paper is focused on the problems of reducing losses of resources at the start-up of new automated factories caused by human shortcomings in teamwork activities. The research is based on a unique database of empirical studies of human faults and mistakes at the design and commissioning of factory automation systems. This database covers over 25 automated factories on three continents, containing pulp and paper mills, chemical and petrochemical plants and power stations. It is shown that the most suitable tool for teamwork analysis is the design structure matrix (DSM)method, which allows visualisation of the full complexity of synergy relations between all team members on the basis of the frequency and amount of information interchange. The mathematical treatment of DSM matrixes enables us to form the most capable teams, to schedule and evaluate their activities. It is shown that as a result of logical analysis it is possible to create an optimal communication and cooperation system, where the competences and capabilities of the teams and their members can be more fully exploited.
\end{abstract}

Key words: Factory automation, engineering design, teamwork management, synergy deployment, quality assurance.

\section{Introduction}

The losses of resources at the start-up of new automated factories caused by human shortcomings reach up to $5 \%-10 \%$ of whole investment costs and tend to increase with the growing complexity of control systems. The real key to reducing these losses is a profound analysis of human shortcomings in the automation systems design and commissioning teamwork.

Nowadays production processes are changing towards higher degrees of automation and new effective solutions in control systems technology. Modern automation systems have been developed to be more universal, being capable of controlling different types of equipment. These requirements also include reconfigure ability, responsiveness and flexibility [1]. In addition to that, advanced functions such as

Corresponding author: Rommi Källo, Ph.D. student, research fields: factory automation, engineering design, synergy and product development. E-mail: rommi.kallo@mail.ee. optimization, scheduling and planning are becoming an important part of process control systems [2]. The traditional barriers between information, communication and automation technologies are, in the operational context, gradually disappearing. The latest technologies, including wireless networks, fieldbus systems and asset management systems, boost the efficiency of automation systems. On the other hand, a specific factory needs a peculiar automation system configured from universal systems [3]. A system can become more complex not only from the engineering side but also due to an increase in the amount of data and variables or the number of disciplines that are involved in the design. Therefore, the need for systems engineering is growing owing to a steady increase in systems complexity.

Despite everything, the key figures in this overall change process are still human beings with their cultural, educational and technical backgrounds and working habits. There search in the field of socio-technical teamwork inefficiency due to human 
shortcomings is a real possibility to reduce the above-mentioned losses in factory automation development and commissioning costs. Experience has shown that human shortcomings can be treated as a result of negative synergy in mutual or inner communication of team members and due to the lack of competence and inability in managing the teamwork in highly competitive environment [4]. Due to the fact that in the development of a multi-agent system the human being is still the key figure, human-related problems must be approached through psychology and teamwork organization. Thus, the management and development of skills and competences in a decentralized organization is becoming increasingly important [3].

From the purely technical side, the reliability of a whole factory automation system also depends on the reliability of the individual components, component interactions and execution environment. Reliability can also be viewed as quality over time [5], where the lifetime of a product or a system is divided into three cycles: running-in, normal work and build-up depreciation. In addition, the impact of faults on reliability differs, depending on how the system is used [6]. So, in reality we meet a complex mix of technical and human factors.

For the better integration of all these matters the synergy-based approach is used in the present research, which aims to decrease the weaknesses and to boost the beneficial features at joining technologies and human activities. The synergy-based approach to the start-up difficulties of factory automation systems is comparatively new, providing an opportunity to analyze the real reasons of human-based start-up problems and to plan the measures to avoid them.

\section{Empirical Studies of Teamwork Quality}

The firm basis of any research in the field of effectiveness of human cooperation is its reality database concerning empirical studies of human shortcomings. The existence of such a unique database ensures the reliability and authenticity of the results attained by theoretical research. The human shortcomings database at the design and commissioning of factory automation systems has been collected by author's team since 1999 .

In the calendar plan, the process of automated factory engineering design covers the drafting of the general description of the system, detailed task description for system configuration and a factory acceptance test, followed by commissioning of the factories as a separate but conjunct part of the database. The human shortcomings in the described above procedures at the beginning of the research up to year 2006 were classified as human faults (misunderstanding between the team members and negligence) and as mistakes (due to lack of competence and absence of special knowledge that can be found only in the process of testing) [4].

For the present research a new and more detailed database for the years 2006-2013 was compiled. The introduced advanced classification of human shortcomings is shown in Fig. 1.

Human shortcomings in this new database are divided into three main categories: faults, mistakes and strategic miscalculations. Technical problems form a separate category. Faults are wrong decisions that have no justification. The faults class F1 includes all misunderstandings in communication between the client, consultant and the design teams or between the

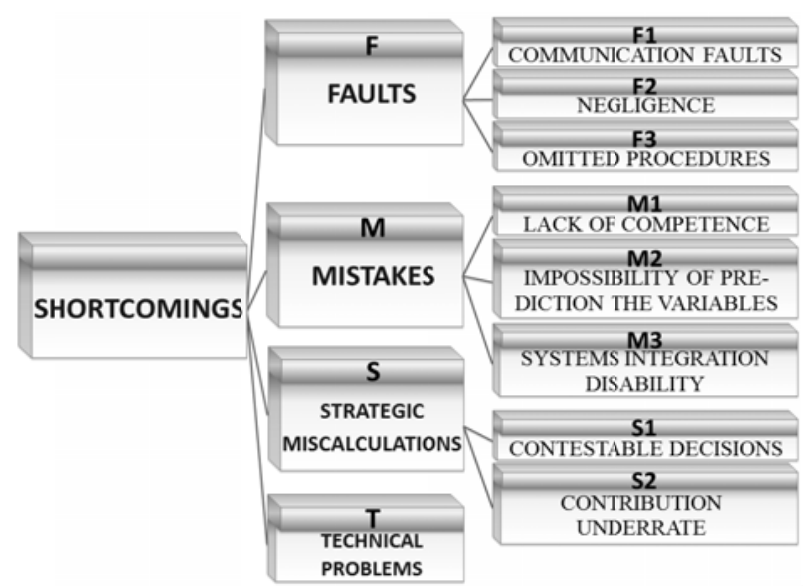

Fig. 1 Advanced classification of human short comings. 
design team members. F2 joins together all shortcomings connected with negligence. All transfers of unsuitable or late information and documentation in the design process are assorted into the faults class F3. The latest covers also faults from information overflow, where lot of e-mails are sent out and the sender predicts that successful communication has occurred.

Mistakes have a far more complicated nature. This category includes wrong decisions M1, caused by lack of core competence. Mistakes M2 are conditional and are caused by the impossibility of predicting the characteristics of the production process at the moment of design and they may be resolved in the course of further projects activities. To the third class M3 belong mistakes caused by system integration disability, which leads to the situation where technologies cannot be integrated due to their different level of development. A new differentiated category of human shortcomings is strategic miscalculations S. Contestable decisions S1 may be taken due to temptation to use cheaper or simple technical solutions, which are not able to grant the necessary operating ability and quality. Underestimation of contribution S2 is a very spread phenomenon in a highly competitive society when under market pressure unreal obligations are accepted. A special category here is that of technical problems $\mathrm{T}$ which involve classical reliability problems.

The real database consists of the information about how the shortcomings were discovered and it may not always describe the real nature of the shortcoming but only its symptoms. Therefore, a thorough analysis of the current situation is often required when a shortcoming has occurred in order to reveal its real reason.

In Fig. 2, the statistics of shortcomings for factory automation systems design and application on the factory acceptance test (FAT) level are presented. For obvious reasons the factories are confidential.

Misunderstandings in communication F1, negligence faults F2 and documentation transfer failures F3 constitute more than $50 \%$ of all short

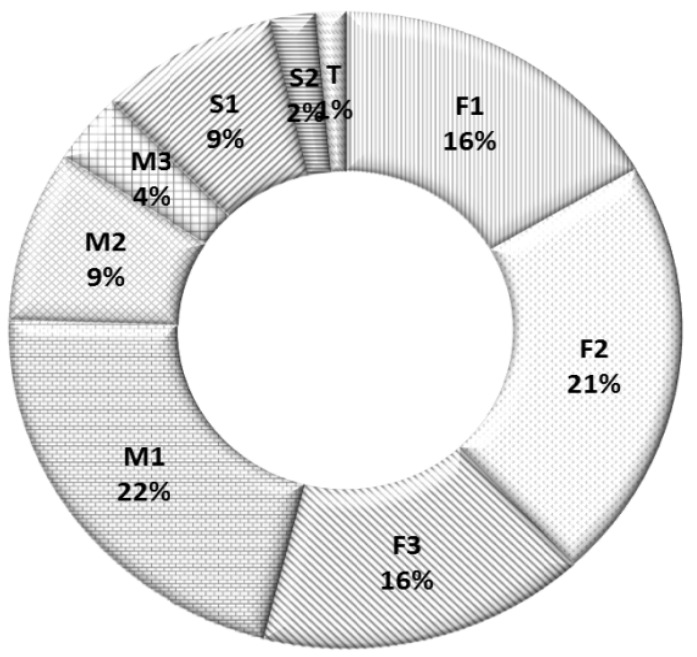

Fig. 2 Statistics of shortcomings for the design of factory automation systems being discovered during the FAT.

comings. These faults are usually clarified during the FAT, but it takes a lot of time and resources. F1 type of faults arise due to lack of communication, where all necessary information is not handed over from one team to another and the latter works out a solution without exact knowledge. This group also includes the items not discussed, when different persons or working groups understand the solutions in different ways. Negligence faults F2 usually comprise mistakes in copying the documents, graphic configurations or application items and also faults due to not reading the documents carefully. Faults F3 cause the biggest resource and time losses as unsuitable information leads to extra work, which easily compromises the time schedule and budget issues. The usual fault in category F3 is missing information in documents or not meeting the document delivery deadlines, which leads to problems with the deployment of manpower and being late.

Mistakes due to lack of necessary competence M1, failures of presaging variables M2 and system integration disability M3 make up $30 \%$ of total shortcomings. Mistakes are more difficult to avoid as individual and overall competence levels have to be identified before the start of the project. Mistakes M1 are caused by lack of team members' personal competence and sometimes they may become evident 
only during the virtual testing of systems. However, the missing competence can be usually found inside the team and the problems can be solved. The M2 types of mistakes are more difficult to discover and fix due to unavailable competence in the specific area. The need for possible modifications may arise only at the design of sub-processes or testing of the configured software. Some variables of processes can be evaluated only during the process of commissioning the new automated factory. The solving of this kind of problems requires a high level of overall competence and flexibility from all the working groups involved and depending on the level of complexity it can be very time-consuming. The system integration problems M3 are mainly related to system hard- and software, where the stage of development of different systems varies and does not always consider all the requirements from the subsystems involved. Incomplete testing of different subsystem integration can lead to significant losses in time and resources as new solutions have to be contrived in a very short time.

Even the quantity of strategic miscalculations $S$ is smaller than faults $\mathrm{F}$ and mistakes $\mathrm{M}$, their impact on the whole project can be significantly bigger as a considerable number of appliances might be involved. Contestable decisions S1 usually occur, when the work packages for certain groups are relatively big and a temptation may arise to increase the profit at the expense of small sub-items. The S2 types of problems are caused by market pressure, when the project-oriented companies are unable to schedule their work effectively. Trying to survive on the market, they need to accept all the orders and hope that somebody else's delay will open up an opportunity for them. The ratio of technical problems $\mathrm{T}$ is trivial and consists mainly of system hardware failures.

In Fig. 3, an analysis of the shortcomings database for the commissioning period of factory automation systems is presented.

The biggest group of shortcomings is again faults $\mathrm{F}$ like during the FAT. However the nature of faults is

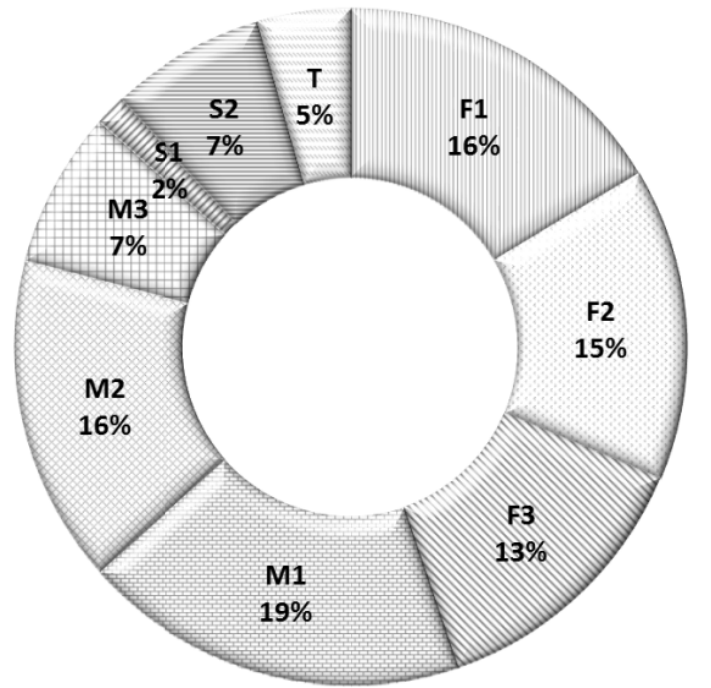

Fig. 3 Statistics of shortcomings for the design of factory automation systems being discovered during commissioning.

different, as their biggest share is formed by communication faults F1. The critical areas for complex factories are the affiliation areas between the systems. The systems themselves are tested during the FAT and operate normally as single units, but the connection suitability can be tested only during commissioning. As new teams are introduced into the project, it is important to ensure the smooth workflow of documentation exchange. The documentation is more exact at this stage and small mistakes rise to the fore and boost. The second substantial group of faults in the commissioning phase is negligence F2, which covers the mistakes during installation, calibration and configuration of field instruments and automation system(s). The fault group F3 of omitted procedures is formed from the designer's inability to consider the entire situation in production systematically. In some cases omitted procedures can indicate even deficiency in Hazard and Operability Study (HAZOP) or safety integrity level (SIL) assessment.

The second, a slightly smaller part of shortcomings falls into the group of misunderstandings $\mathrm{M}$, where the biggest share is caused by lack of competence M1. The know-how about complicated subsystems is becoming increasingly important and in this context only the overall knowledge about the process is not enough 
anymore. The process equipment designed and delivered by different companies lacks interoperability experience. Sometimes it is impossible to predict all the situations and concurrence of separate subsystems (mistakes M2).As the number of variables is large, it is not realistic to evaluate and adjust them before commissioning. In the rapidly developing technology world there is always a risk to run into systems that are at different levels of development. Compatibility problems constitute the majority of faults M3 (systems integration disability).

A smaller but more time- and resources-consuming group of shortcomings in the commissioning phase is the strategic miscalculations S. Due to the market pressure companies try to take a bigger portion of work than the knowledge and experience would allow. As it is difficult to assess each supplier's capability to perform single work operations, the structure of the team can change during work progress. The enforcement of operations by management and financial people usually not tolerating the resignation, leads to difficult situations in the commissioning process, where the final solution due to the miscalculation made in the beginning, can be expensive and time-consuming.

The technical faults $\mathrm{T}$ in the commissioning phase are mainly caused by transportation damage, the unsuitable type of equipment or faulty communication interface between the subsystems.

\section{Deployment of Synergy Dependencies in Teamwork}

The main goal of the synergy-based analysis of multi-agent automation systems is to find a design and commissioning principles that would be able to highlight and amplify positive synergy and prevent mismatches caused by the negative synergy of technologies and in teamwork. The search for a powerful tool for describing human relations and grouping them on the basis of their cooperation capabilities has proved that the most suitable tool for that is the design structure matrix (DSM) modelling technique that allows visualizing the full complexity of the relations between all team members [7].

Fig. 4 presents a matrix of personal activities during the design of a typical factory automation system

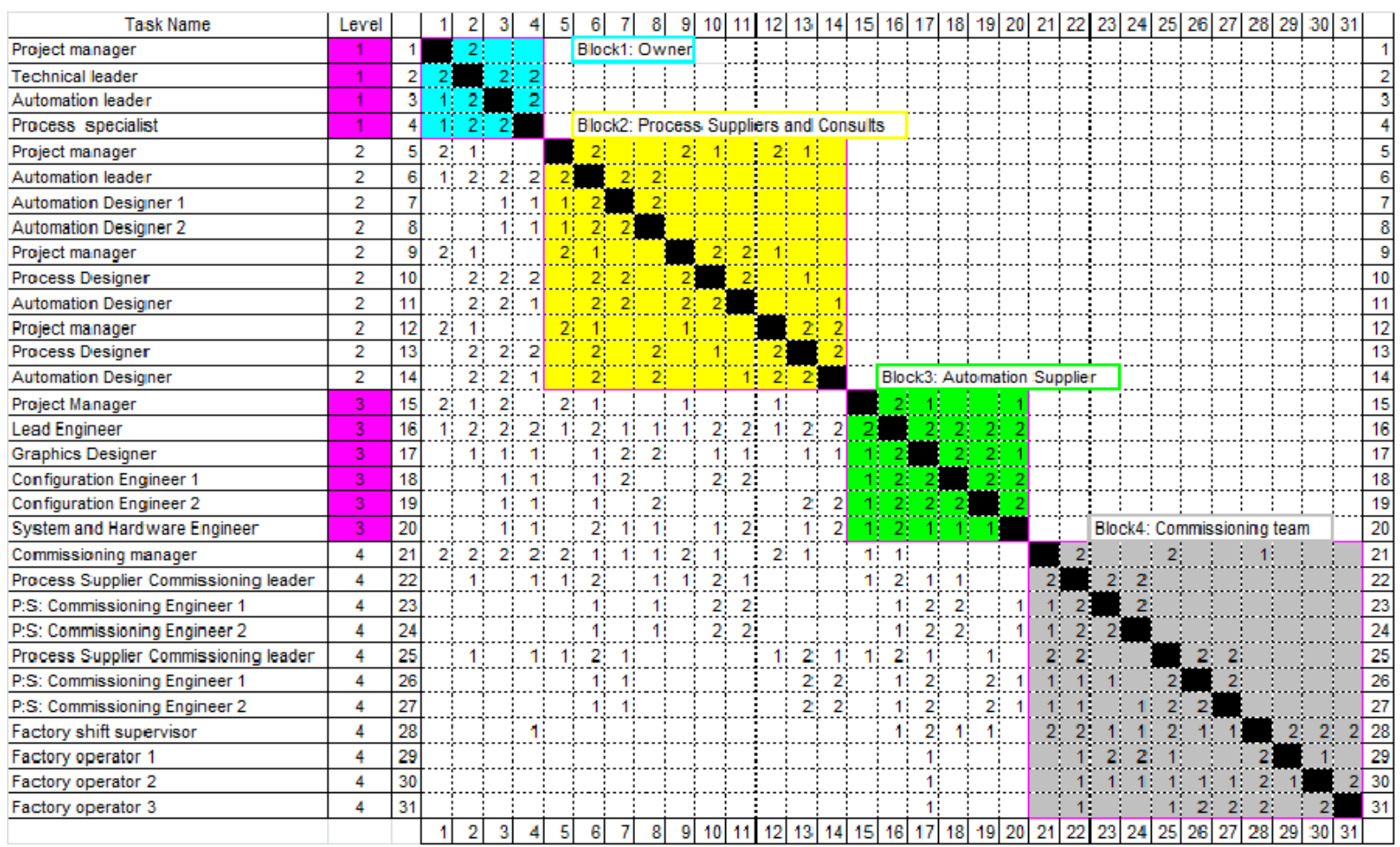

Fig. 4 The DSM of personal relations in teamwork. To previous page. 
project ending with the launch of the factory. First of all, the owner names responsible persons in his team to keep watch on the progress of building a new production plant and to transfer his own requirements and necessary competence from the already existing production. Next, to run the project a consultant company is hired to integrate all efforts of project and commissioning groups. The task of the consultancy group is to forward the owner's requirements to the process supplier(s). After that, it is necessary to collect the required data from the process supplier(s), convert them to the required format, get approval from the owner and give this input information to the automation supplier. Then the automation supplier starts system configuration, including application software programming, human-machine interface and process interfaces configuration. After the configuration is complete and the automation system is tested in the automation supplier's workshop, it is delivered to the factory and integrated with the process supplier's equipment. The activities continue with commissioning, where a lot of new people will be involved in the project team. A separate commissioning team is formed, which includes members from project teams. The end users also participate in the commissioning, getting trained to run the plant at the same time.

At compiling the matrix, all inputs/persons are preliminarily numbered in order to involve synergy relations between persons in the matrix. Therefore, the numbers of inputs must be the same on vertical and horizontal axes. The number of inputs is practically not limited and depends only on the complexity of the project. It is comparatively easy to introduce the synergy dimension into the matrixes to empower teamwork capability through the evaluation of synergy coactions between persons involved. The strength of the coaction can be characterised on a 3-step scale: (0) indifferent (left blank), (1) coactionis moderate and (2) coaction is strong. Here, the direction of coaction is very important and in case the results of the previously completed task are used in the following one, only the first should be written into the matrix. If the information flow is from the top down, the subdiagonal part of the matrix is filled. The super diagonal part is filled when the information from the group member below is needed to complete the task. The specific feature of the used DSM model is its bigger flexibility and universality for describing real communication processes It was already successfully used at creating tools for interdisciplinary systems design and their quality assurance $[8,9]$. The mathematical treatment of matrixes enables to form the most capable teams, to schedule their activities and predict the time necessary to complete the tasks, considering the probable iterations and reworks [10, 11].

The practical use of the matrix for growing synergy in teamwork depends mainly on the selected scope of the matrix information. There are 3 ways of using the collected data for empowering cross-team activities. The first way is a logical analysis of human shortcomings and looking for possibilities of better communication and cooperation. The second way is to use the resources of the DSM method for the same purpose. And the third way is to use soft computing tools to find synergistic solutions for cross-teamwork. All the listed ways are simultaneously important for the research group but in the present paper the results of the first approach are mainly described.

When taking a look at (Fig. 4) the whole matrix, it is useful to consider the following recommendations. The mathematical processing of the matrix shows that it is impossible to treat automation and process design groups as separate levels of the matrix but it is necessary to integrate them into one team, even if they come from different companies. Exactly the same applies to commissioning engineers and factory personnel. So the people involved in commissioning should not be focused on the company they are working for, but the team, which is formed from all the participants in the commissioning process. At the same time the leader of the group of process design and 
commissioning should treat the engineers as group members, irrespective of organisational and financial aspects. Following the recommendations of the DSM matrix, for example, it is possible to mitigate the faults $\mathrm{F}$ by setting the communication procedures and sharing the responsibilities. Also, personal motivation is important. Although more difficult, it is possible to obviate the mistakes $\mathrm{M}$ by training and upgrading the personnel and involving qualified and experienced consultants in the teams. The strategic miscalculations $\mathrm{S}$ can only be minimized during business negotiations specifying the needs very precisely and stipulating the project plan on a detailed level.

If the scope is addressed to the concrete working group, teamwork interactions dominate. Building synergy inside the team starts with the establishing of hierarchy structure and clear tasks on every level. The structure of communication inside the teams is highly interdependent as it is shown in Fig. 4. Financial and technical tasks and rules must be very clearly defined. The overall scheduling of the work is important as the next group can start their work after the previous has finished their task. However, scheduling does not exclude later clarification of the task or even modifications caused by new knowledge obtained during the project, but these activities cannot be fixed in the matrix. The communication between the groups also needs to be scheduled and organised to ensure the smooth progress of the project. The last scope is personal relations. In this sphere competence dominates in parallel with psychological and cognitive dimensions.

\section{Conclusions}

The main value of the present research is aunique database of empirical studies of human shortcomings in teamwork at the design and commissioning of factory automation systems. As the majority of start-up difficulties are related to human beings with their imperfection, teamwork is the most important entity to analyze and improve. The analysis of this database opens the way to reduce the losses of resources at the start-up of new automated factories.

The design structure matrix (DSM) technology is a capable tool for visualizing synergy relations between development projects working groups in the information interchange during group teamwork and in group members' personal relations. The DSM technology enables to form the most capable teams, to schedule their activities and predict the time necessary to complete the project. In the final part of the paper recommendations are worked out for building up a synergy-based communication system to fully exploit the teams' capabilities and their members' competence.

The future processing of the data by tools of soft computing allows finding ways of increasing synergy in teamwork and substantially reducing the losses of starting up new factories.

\section{References}

[1] I. Seilonen, I. Pirtioja, K. Koskinen, Extending process automation systems with multi-agent techniques, Engineering Applications of Artificial Intelligence 22 (2009) 1056-1067.

[2] T. Samad, P. McLaughlin, J. Lu, System architecture for process automation: Review and trends, Journal of Process Control 17 (2007) 191-201.

[3] S.-L. Jämsä-Jounela, Future trends in Process process Automationautomation, Annual Reviews reviews in Controlcontrol 31 (2007) 211-220.

[4] F. Kaljas, R. Källo, V. Reedik, Human aspects at design of mechatronic systems, in: Proceedings of the 9th Mechatronic Forum International Conference, Ankara, Turkey, Atilim University Publications, 2004, pp. 147-157.

[5] J.-W. Sun, L.F. Xi, S.C. Du, B. Ju, Reliability modeling and analysis of serial-parallel hybrid multi-operational manufacturing system considering dimensional quality, tool degradation and system configuration, Int. J. Production Economics 114 (2008) 149-164.

[6] M. Palviainen, A. Evesti, E. Ovaska, The reliability estimation, prediction and measuring of component-based software, The Journal of Systems and Software 84 (2011) 1054-1070.

[7] S.D. Eppinger, T. Browning, Design Structure sructure 
Matrix matrix Methods methods and Applicationsapplications, Massachusetts Institute of Technology, Cambridge, MA, USA, 2012.

[8] F. Kaljas, Synergy-based approach to design of the interdisciplinary systems, Ph.D. Thesis, Mechanical and Instrumental Engineering E24, Tallinn University of Technology, TUT Press, 2005.

[9] T. Hindreus, V. Reedik, Synergy-based approach to quality assurance, Estonian Journal of Engineering 15 (2)
(2009) 87-98.

[10] T. Browning, Modeling and analyzing cost, schedule and performance in complex system product development, Ph.D. Thesis, Massachusetts Institute of Technology, Cambridge, MA, 1998.

[11] S.H. Cho, S.D. Eppinger, Product development process modelling using advanced simulation, in: Proceedings of ASME Design Engineering Technical Conference DETC’01,Pittsburg, Pennsylvania, USA, 2001, pp. 1-10. 